\title{
Modelo de Ganho de Canal em Comunicações Moleculares para um Sistema Circulatório de Gestantes
}

\author{
Carlos Danilo Miranga Regis, Catarina Lenice Lopes Pedrosa, Iasmin de França Albuquerque, Michael Taynnan \\ Barros, Walisson da Silva Soares
}

\begin{abstract}
Resumo-A gestação é um período no qual o corpo e o sistema imunológico da mulher sofrem alterações progressivas, resultando em alterações hemodinâmicas características deste período. Esses efeitos afetam sistemas de comunicações moleculares sanguíneos, e mudam a propagação de sinais em artérias e arvores de artérias. $O$ objetivo deste artigo é implementar um modelo matemático que permita que sejam calculados os valores de fluxo sanguíneo no período gestacional, levando em consideração os novos parâmetros obtidos pelas mudanças ocorridas durante a gestação. Com uma análise de ganho do canal molecular, fora observado que o fluxo sanguíneo pode aumentar até em $\mathbf{8 5 , 5 8 \%}$ para mulheres grávidas e atingir um ganho de $2 \mathrm{~dB}$ do sinal transmitido.
\end{abstract}

Palavras-Chave-Comunicações Moleculares, Gravidez, Hemodinâmicas, Ganho, Fluxo Sanguíneo.

Abstract-Gestation is a period in which the body and the immune system of women undergo progressive changes, resulting in hemodynamic changes characteristic of this period. This phenomenos can affect circulatory molecular communication systems with changes in the propagation of signals in arteries and artery trees. In this paper we present a mathematical model that allows the calculation of the blood flow values during the gestational period, taking into account the new parameters obtained by the changes that occurred during pregnancy. We perform an analysis of the molecular channel gain, and we observed that the blood flow can increase $85,58 \%$ for pregnant women and the gain of the channel can get to a total of $2 \mathrm{~dB}$.

Keywords-Molecular Communications, Pregnancy, Hemodynamics, Gain, Blood Flow.

\section{INTRODUÇÃO}

O novo paradigma das comunicações, Comunicações Moleculares, vêm atraindo pesquisadores com o objetivo de desenvolver uma tecnologia que permita o sensoriamento e atuação em sistemas biológicos, e também, dentro do corpo humano [1]. Inclusos na variedade de tipos desses sistemas propostos pela literatura, existem sistemas que codificam informação pela modulação da concentração de cálcio em tecidos [2], [3], modulação da atividade neuronal [4], e também, modulação de concentração de moléculas de sangue, e outras, dentro do sistema circulatório humano [5]. A maior aplicação de comunicações moleculares nesse contexto é a Entrega Inteligente de Medicamentos, que promete fazer uma transformação na medicina e indústria farmacêutica por adaptar o tratamento de doenças estudando o sistema de comunicações moleculares do corpo sanguíneo e sua caracterização.
Durante a gravidez, a mulher passa por diversas mudanças anatômicas e fisiológicas significativas, para nutrir e acomodar o feto em desenvolvimento. Essas mudanças começam após a concepção e afetam cada sistema de órgãos no corpo. O sistema cardiovascular também sofre alterações progressivas durante a gestação e o parto, resultando em alterações hemodinâmicas características deste período. As mudanças principais envolvem o aumento do volume sanguíneo, do débito cardíaco, a diminuição da resistência vascular sistêmica e da reatividade vascular. $\mathrm{O}$ aumento do volume sanguíneo induzido pela gestação é uma adaptação do organismo materno com o objetivo de suprir a demanda do útero hipervascularizado, bem como para proteger a mãe da perda sanguínea no momento do parto.

O débito cardíaco aumenta progressivamente durante a gestação, começando a se elevar entre a décima e a décima segunda semana de gestação. Este aumento se deve à elevação do volume sistólico secundário, ao aumento do volume sanguíneo e da frequência cardíaca [6]. A frequência cardíaca aumenta e o aumento do débito cardíaco implicará no aumento do fluxo sanguíneo prioritariamente em alguns órgãos, como o útero e a placenta. Essas características alteram a propagação de moléculas, em geral, na corrente sanguínea. Modelos matemáticos devem ser estudados para habilitar um nível de detalhamento maior sobre esses fenômenos, como também, permitir a possibilidade de inferência de estados futuros desse canal.

Com as Comunicações Moleculares aplicadas ao estudo do comportamento dos processos biológicos humanos, a construção de modelos computacionais fazem um papel fundamental em novas aplicações da nanotecnologia para diagnóstico e tratamento de seres humanos. Um sistema de comunicação molecular, normalmente, é dividido em 3 blocos, como mostrado na Fig. 1, quais sejam: nanomáquina transmissora, canal molecular e nanomáquina receptora.

A nanomáquina transmissora é responsável pela codificação da informação em moléculas, e pela sua posterior liberação no canal molecular, que é o meio pelo qual as moléculas de informação se propagam até chegarem à nanomáquina receptora, responsável pela captura das moléculas de informação e sua posterior decodificação.

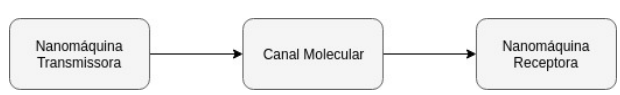

Fig. 1. Sistema de Comunicação Molecular.
526 
O objetivo deste trabalho é fazer um modelo matemático do mapa das artérias humanas para que essa rede arterial possa ser estudada como um canal molecular de um sistema que tem como nanomáquina receptora a placenta de uma mulher grávida. A partir de um modelo matemático determina-se como as artérias são modeladas durante o período gestacional e não-gestacional, levando em consideração as mudanças geradas neste período, bem como calcular como o fluxo sanguíneo se distribui pelo corpo humano até chegar nas artérias que se ligam à placenta, e comparada com os resultados obtidos para uma mulher grávida e não grávida. Fora observado que o fluxo sanguíneo pode aumentar até em $85,58 \%$ para mulheres grávidas nas proximidades da placenta e atingir um ganho de $2 \mathrm{~dB}$ do sinal transmitido. Esses resultados são importantes para futuro desenvolvimento de medicamentos especificamente para mulheres grávidas levando em conta a diferença do ganho do canal que fora observado.

\section{ARtÉRIAS}

As artérias são organizadas em uma sofisticada rede que cobre todo o organismo, chamada de árvore arterial, sendo dividida em artérias grandes e pequenas [5]. Nesta seção, apresenta-se como as artérias foram modeladas de acordo com o modelo de árvore arterial apresentado na Fig. 2, que demonstra a configuração de artérias grandes e suas respectivas terminações em ramos de diversas artérias pequenas [7]. A pressão harmônica $P_{l}\left(\omega_{k}\right)$ e o fluxo harmônico $Q_{l}\left(\omega_{k}\right)$ em uma artéria $l$ podem ser relacionados pela impedância de carga $Z_{l}\left(\omega_{k}\right)$ como mostrado a seguir, na Equação 1, similar a Lei de Ohm se a pressão for considerada como a tensão e o fluxo como a corrente.

$$
P_{l}\left(\omega_{k}\right)=Q_{l}\left(\omega_{k}\right) Z_{l}\left(\omega_{k}\right)
$$

\section{A. Artérias pequenas}

1) Impedância de Carga de uma Artéria Pequena: O modelo de uma artéria pequena $l$ é explicado como um componente elétrico com uma impedância de carga $Z_{l}\left(\omega_{k}\right)$.

As artérias pequenas possuem parâmetros de escala $\alpha$ e $\beta$, que relacionam o raio de duas artérias bifurcadas: a da esquerda $r_{l}$ e a da direita $r_{l^{\prime}}$ com o raio de sua artéria mãe $\left(r_{l-1}\right)\left(r_{l}=\alpha r_{l-1}\right.$ e $\left.r_{l}^{\prime}=\beta r_{l-1}\right)$.

A impedância harmônica na entrada da artéria pequena $Z_{l}\left(\omega_{k}\right)$ e em sua saída $Z_{l}^{\text {out }}\left(\omega_{k}\right)$ são relacionadas por [5]:

$$
Z_{l}\left(\omega_{k}\right)=\frac{j \tau_{l}\left(\omega_{k}\right) \sin \left(\tau\left(\omega_{k}\right)\right) / \ell_{l}+Z_{l}^{\text {out }}\left(\omega_{k}\right) \cos \left(\tau_{l}\left(\omega_{k}\right)\right)}{\cos \left(\tau_{l}\left(\omega_{k}\right)\right)+j \ell_{l} Z_{l}^{\text {out }}\left(\omega_{k}\right) \sin \left(\tau_{l}\left(\omega_{k}\right)\right) / \tau_{l}\left(\omega_{k}\right)}
$$

em que:

$$
\tau_{l}\left(\omega_{k}\right)=\frac{l_{r r}}{\sqrt{\pi}} \sqrt{\frac{\rho c_{l}}{1-\frac{2 J_{1}\left(j r_{l}^{2} \omega / \nu\right)}{j r_{l}^{2} \omega / \nu J_{0}\left(j r_{l}^{2} \omega / \nu\right)}}} .
$$

Considerando que $r_{l}$ é o raio da artéria pequena, $\ell_{l}$ é o comprimento da artéria pequena e $l_{r r}$ é a relação comprimento/raio, $\rho$ é a densidade do sangue, $\nu$ é a viscosidade do sangue, $c_{l}$ é o volume da artéria pequena, $J_{0}$ e $J_{1}$ são as funções de Bessel, de ordem zero e primeira ordem, respectivamente [8].

Para um valor de $\omega_{k}$ constante, a partir da Equação 2, é possível obter:

$$
Z_{l}\left(\omega_{k}\right)=\frac{8 \mu \ell_{l}}{\pi r_{l}^{4}+Z_{l}^{\text {out }}\left(\omega_{k}\right)}
$$

A conservação do fluxo na bifurcação e a continuidade de pressão justifica a modelagem de bifurcações como ramos de fios condutores perfeitos na analogia elétrica do fluxo de sangue e pressão, e permite a aplicação das leis das correntes e tensões de Kirchoff. A impedância harmônica na saída $Z_{l}^{\text {out }}\left(\omega_{k}\right)$ pode ser relacionada com a impedância harmônica da artéria $(l+1) Z_{l+1}\left(\omega_{k}\right)$ e a impedância harmônica de sua irmã $Z_{l^{\prime}+1}$ pela relação a seguir:

$$
Z_{l}^{\text {out }}\left(\omega_{k}\right)=\left(\frac{1}{Z_{l+1}\left(\omega_{k}\right)}+\frac{1}{Z_{l^{\prime}+1}\left(\omega_{k}\right)}\right)^{-1}
$$

A árvore de artérias pequenas é truncada quando o raio $r_{l}$ é menor que um determinado $r_{\text {min }}$, pois a impedância harmônica de uma artéria pequena $l$ com $r_{l}<r_{\min }$ é considerada zero.

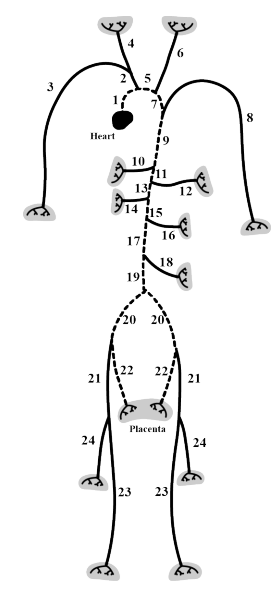

Fig. 2. Modelo de Rede Arterial.

\section{B. Artérias Grandes}

1) Matriz de Transferência e Impedância: O fluxo de sangue em uma artéria grande é assumido como laminar, viscoso e incompressível, e essa pressão é constante sobre sua seção transversal [7]. Ao aplicar a análise do circuito de rede quadripolo, é possível relacionar o fluxo de entrada $Q_{i n}\left(\omega_{k}\right)$ e a pressão de entrada $P_{i n}\left(\omega_{k}\right)$ com o fluxo $Q_{l}\left(\omega_{k}\right)$ e a pressão $P_{l}\left(\omega_{k}\right)$ em uma determinada artéria $l$ através da matriz de transferência $T\left(\omega_{k}\right)$, como mostra a Equação 6.

$$
\left[\begin{array}{c}
P_{i n}\left(\omega_{k}\right) \\
Q_{i n}\left(\omega_{k}\right)
\end{array}\right]=\mathbf{T}\left(\omega_{k}\right)\left[\begin{array}{c}
P_{l}\left(\omega_{k}\right) \\
Q_{l}\left(\omega_{k}\right)
\end{array}\right]
$$

Em que: 
Os coeficientes da Equação 7 são elementos da matriz de transferência $T\left(\omega_{k}\right)$ da artéria grande $l$ e são definidos como:

$$
\begin{gathered}
A\left(\omega_{k}\right)=\cosh \left(\gamma_{l}\left(\omega_{k}\right) \ell_{l}\right), \\
B\left(\omega_{k}\right)=Z_{l}^{\circ}\left(\omega_{k}\right) \sinh \left(\gamma_{l}\left(\omega_{k}\right) \ell_{l}\right), \\
C\left(\omega_{k}\right)=\frac{1}{Z_{l}^{\circ}\left(\omega_{k}\right)} \sinh \left(\gamma_{l}\left(\omega_{k}\right) \ell_{l}\right), \\
D\left(\omega_{k}\right)=\cosh \left(\gamma_{l}\left(\omega_{k}\right) \ell_{l}\right),
\end{gathered}
$$

na qual o $\gamma_{l}\left(\omega_{k}\right)$ é o coeficiente de propagação do segmento da artéria grande $l, Z_{l}^{\circ}\left(\omega_{k}\right)$ é a impedância característica da artéria e $\ell_{l}$ é o seu comprimento.

Além disso, a matriz de transferência $T\left(\omega_{k}\right)$ para uma artéria grande $l$ depende de suas dimensões geométricas e parâmetros fisiológicos da artérias, que são os seguintes:

a) Raio de afunilamento: uma artéria grande $l$ é considerada como um tubo axissimétrico como o ilustrado na Fig. 3, em relação ao raio decrescente e o comprimento $l$. A entrada da artéria grande $l$ tem um raio superior $r_{l}^{\text {top }}$ e sua saída possui um raio inferior $r_{l}^{b o t}$. Os valores numéricos da equação são encontrados a partir de medidas anatômicas de acordo com a Tabela I. Considerase $z$ como a coordenada de longitude ao longo do eixo da artéria grande, o raio $r_{l}(z)$ diminui exponencialmente de $r_{l}^{\text {top }}$ em $z=0$ a $r_{m}^{b o t}$ em $z=\ell_{l}$ de acordo com a Equação 9 , na qual $l_{l}$ é o fator de afunilamento para a artéria grande $l$, definido na Equação 10 [7].

$$
\begin{gathered}
r_{l}(z)=r_{l}^{t o p} \exp \left(-k_{l} z\right) \\
k_{l}=\frac{r_{l}^{t o p} / r_{l}^{b o t}}{l_{\ell}}
\end{gathered}
$$

b) A conformidade do volume quantifica a tendência das paredes da artéria a produzir pressão e outras forças externas. A partir de estudos estatísticos de medidas fisiológicas [5], a conformidade do volume $c_{l}$ pode ser estimada por:

$$
c_{l}(z)=\frac{\pi r_{l}^{2}(z)}{k_{1} \exp \left(-k_{2} r_{l}(z)\right)+k_{3}}
$$

Os valores das constates são: $k_{1}=1.34 \times 10^{7} \mathrm{~g} /\left(\mathrm{s}^{2} . \mathrm{cm}\right)$, $k_{2}=22.53 \mathrm{~cm}^{-1}$ e $k_{3}=5.77 \times 10^{5} \mathrm{~g} /\left(\mathrm{s}^{2} \mathrm{~cm}\right)$.

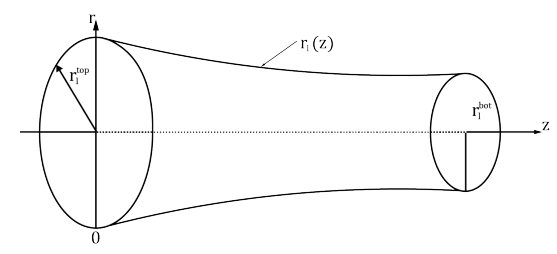

Fig. 3. Artéria grande $m$ como um tubo axissimétrico com raio de afunilamento no plano $(r, z)$.

A impedância de carga $Z_{l}^{\circ}\left(\omega_{k}\right)$ é uma medida de oposição experimentada pelo fluxo sanguíneo na entrada de uma artéria grande $l$. Ela depende da topologia de todas as artérias
TABELA I

VALORES DE COMPRIMENTOS E RAIOS DA ÁRVORE ARTERIAL [7].

\begin{tabular}{c|c|c|c|c}
\hline $\mathrm{n}^{\mathrm{a}}$ & Artéria & $l(\mathrm{~cm})$ & $r_{\text {top }}(\mathrm{cm})$ & $r_{\text {bot }}(\mathrm{cm})$ \\
\hline 01 & Aorta Ascendente & 7,00 & 1,25 & 1,14 \\
02 & Braquiocefálica & 3,50 & 0,70 & 0,70 \\
03,08 & Subclávia e Braquial & 43,00 & 0,44 & 0,28 \\
04 & Carótida D. & 17,0 & 0,29 & 0,28 \\
05 & Arco da Aorta D. & 1,80 & 1,14 & 1,11 \\
06 & Carótida E. & 19,0 & 0,29 & 0,28 \\
07 & Arco da Aorta E. & 1,00 & 1,11 & 1,09 \\
09 & Torácica & 18,8 & 1,09 & 0,85 \\
10 & Celíaca & 3,00 & 0,33 & 0,30 \\
11 & Aorta Abdominal & 2,00 & 0,85 & 0,83 \\
12 & Mesentérica Sup. & 5,00 & 0,33 & 0,33 \\
13 & Aorta Abdominal & 2,00 & 0,83 & 0,80 \\
14,16 & Renal & 3,00 & 0,28 & 0,25 \\
15 & Aorta Abdominal & 1,00 & 0,80 & 0,79 \\
17 & Aorta Abdominal & 6,00 & 0,70 & 0,73 \\
18 & Mesentérica Inf. & 4,00 & 0,20 & 0,18 \\
19 & Aorta Abdominal & 3,00 & 0,73 & 0,70 \\
20 & Ilíaca Externa & 6,50 & 0,45 & 0,43 \\
21 & Femoral & 13,0 & 0,43 & 0,40 \\
22 & Ilíaca Interna & 4,50 & 0,20 & 0,20 \\
23 & Femoral Profunda & 11,0 & 0,20 & 0,20 \\
24 & Femoral & 44,0 & 0,40 & 0,30 \\
\hline \multicolumn{4}{r}{}
\end{tabular}

grandes que se ramificam da grande artéria $l$ e suas dimensões geométricas.

Usando a teoria de linhas de transmissão [9], é possível expressar a impedância de carga da artéria grande $l$ em função da impedância de carga em sua saída, que é denotada $Z_{l}^{\text {out }}\left(\omega_{k}\right)$ como mostra a Equação 12.

$$
Z_{l}\left(\omega_{k}\right)=Z_{l}^{\circ}\left(\omega_{k}\right) \frac{Z_{l}^{\text {out }}\left(\omega_{k}\right)+Z_{l}^{\circ}\left(\omega_{k}\right) \tanh \left(\gamma_{l}\left(\omega_{k}\right) \ell_{l}\right)}{Z_{l}^{\circ}\left(\omega_{k}\right)+Z_{l}^{\text {out }}\left(\omega_{k}\right) \tanh \left(\gamma_{l}\left(\omega_{k}\right) \ell_{l}\right)}
$$

O $Z_{l}\left(\omega_{k}\right)$ e $\gamma_{l}\left(\omega_{k}\right)$ são, respectivamente, a impedância característica e o coeficiente de propagação para a artéria grande $l\left(\omega_{k}\right)$. Se a artéria grande divide-se em outras duas artérias grandes, a impedância de carga na saída é dada pela Equação 5, da mesma forma que foi apresentada na secção anterior.

Caso contrário, a artéria é terminada por uma árvore de pequenas artérias e a impedância de carga na saída da artéria grande é exatamente a impedância de carga da interface com as artérias pequenas.

\section{MODELO HEMODINÂMICO PARA O PERÍODO DA GRAVIDEZ}

Durante a gravidez, todo o sistema cardiovascular é remodelado para acomodar as necessidades fetais, apesar do aumento da carga de trabalho do coração durante a gestação e o trabalho de parto, a mulher saudável não apresenta comprometimento da reserva cardíaca. O volume sanguíneo aumenta, mais vasos sanguíneos crescem e a pressão do útero em expansão em grandes veias faz com que o sangue retarde seu retorno ao coração. O débito cardíaco começa a se elevar entre a décima e a décima segunda semana de gestação, atingindo $30 \%$ a $50 \%$ em relação aos níveis pré-gravídicos até a trigésima segunda semana de gestação [6]. Este aumento se deve à elevação do volume sistólico secundário, ao aumento do volume sanguíneo 528 
e da frequência cardíaca. O volume sanguíneo ejetado pelo coração, débito cardíaco, pode ser representado pela relação apresentada em $D c=V s \times F c$, em que, $D c$ é o débito cardíaco, $V s$ é o volume sistólico e $F c$ representa a frequência cardíaca.

A frequência cardíaca aumenta de dez a quinze batimentos por minuto durante a gestação. $\mathrm{O}$ aumento do débito cardíaco implicará na maior perfusão em alguns órgãos, como do útero e da placenta, ampliando progressivamente durante a gestação o aporte sanguíneo para o feto em crescimento, dos rins, cujo suprimento sanguíneo aumenta em aproximadamente $500 \mathrm{ml} / \mathrm{min}$, da pele, para eliminação mais eficaz do calor, das glândulas mamárias, intestinos entre outros. A resistência vascular é definida como a relação da pressão com o fluxo arterial, apresentada na Equação 1. A diminuição da resistência vascular sistêmica está associada, em parte, ao grande leito vascular uteroplacentário. Esta diminuição é máxima por volta da segunda metade da gestação, fase em que ocorre a segunda onda de migração trofoblástica. Neste momento, há invasão das artérias espiraladas do útero pelo tecido trofoblástico, fazendo com as mesmas percam sua camada muscular [6].

A Tabela II apresenta resumidamente as mudanças direcionais que são bem caracterizadas na gravidez [10].

TABELA II

Mudanças Hemodinâmicas na Gravidez.

\begin{tabular}{c|c}
\hline Aumento & Decréscimo \\
\hline Fluxo Sanguíneo & Resistência Vascular Sistêmica \\
\hline Volume Plasmático & Resistência Vascular Pulmonar \\
\hline Frequência Cardíaca & Pressão Arterial Sanguínea \\
\hline Capacitância Venosa & Pressão osmótica coloidal \\
\hline
\end{tabular}

\section{MÉtodos}

Para realizar o cálculo dos fluxos sanguíneos em cada artéria do corpo foi considerado um sinal de entrada modelado de acordo com o da Fig. 4, em seguida foram efetuados os cálculos dos raios médios de cada artéria, mapeadas como apresentado na Fig. 2, utilizando os parâmetros de raio inferior, superior e comprimento de cada artéria em questão, apresentados na Tabela I.

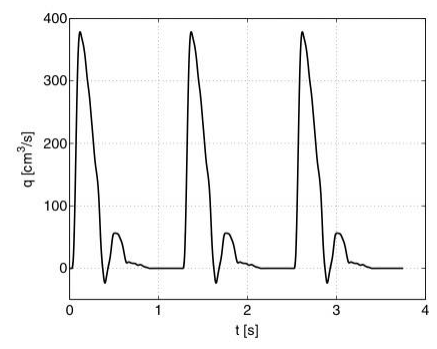

Fig. 4. A entrada em função do tempo com comprimento de três ciclos cardíacos [7].

Os valores de impedância característica e os parâmetros da matriz de transferência foram calculados de acordo com a fundamentação apresentada na Secção II. O cálculo das impedâncias de carga equivalentes foi realizado de acordo com a configuração da arvore arterial, sendo a Equação 12 utilizada nas artérias que se bifurcam em duas outras artérias grandes, e um algoritmo recursivo para as que terminam em uma árvore de artérias pequenas, utilizando os fatores de assimetria $\alpha$ e $\beta$ como 0,9 e 0,8 respectivamente [5]. Assim, para realizar os cálculos de cada período (pré-gravídico e durante os três primeiros bimestres da gestação) foi necessário considerar as mudanças nos parâmetros hemodinâmicos apresentados na Secção III e numericamente apresentados na Tabela III, que apresenta os valores dos parâmetros utilizados antes da gravidez, após 8, 16 e 24 semanas de gestação. Por fim, foram realizados os cálculos de fluxo nas principais artérias do corpo, analisando o comportamento do fluxo sanguíneo do coração até a placenta para cada período avaliado.

TABELA III

Medições do ParÂMetros CARdiovasculares.

\begin{tabular}{c|c|c|c|c}
\cline { 2 - 5 } & $\begin{array}{c}\text { Anterior } \\
\text { a gestação }\end{array}$ & $\begin{array}{c}8 \\
\text { semanas }\end{array}$ & $\begin{array}{c}16 \\
\text { semanas }\end{array}$ & $\begin{array}{c}24 \\
\text { semanas }\end{array}$ \\
\hline $\begin{array}{c}\text { Frequência } \\
\text { Cardíaca (bpm) }\end{array}$ & 65,00 & 68,00 & 72,00 & 73,00 \\
\hline $\begin{array}{c}\text { Fluxo } \\
\text { Sanguíneo }\left(\mathrm{cm}^{3} / \mathrm{s}\right)\end{array}$ & 70,00 & 86,67 & 98,33 & 95,00 \\
\hline $\left.\begin{array}{c}\text { Resistência } \\
\text { Vascular (ds/cm }\end{array}\right)$ & 1376,00 & 969,00 & 926,00 & 930,00 \\
\hline $\begin{array}{c}\text { Pressão } \\
\text { Sanguínea (mmHg) }\end{array}$ & 69,00 & 62,00 & 68,00 & 67,00 \\
\hline
\end{tabular}

Foi calculado o ganho do canal, relacionando os valores máximos de fluxos sanguíneos dos quatro períodos analisados, sendo o pré-gravídico utilizado como referência. Para fins deste trabalho, foi levado em consideração apenas o caminho percorrido pelo fluxo sanguíneo entre o coração e a placenta. A equação a seguir apresenta o ganho em $d B$.

$$
G_{k}=10 \log \left(\frac{Q_{l k}}{Q_{l}}\right)
$$

Em que, $Q_{l k}$ é o fluxo sanguíneo, com $k=\{1,2,3\}$ representando os três primeiros bimestres da gestação e $Q_{l}$ é o fluxo no período pré-gravídico.

\section{Resultados}

O comportamento do fluxo sanguíneo foi analisado para 4 períodos distintos, o pré-gravídico bem como os três primeiros bimestres gestacionais. Dessa maneira, a Fig. 5(a) apresenta o comportamento do fluxo pelo tempo na aorta ascendente, na qual as quarto curvas correspondem a cada período citado.

$\mathrm{Na}$ aorta ascendente houve um aumento percentual no fluxo de aproximadamente $27 \%$ na oitava semana de gestação, já na décima sexta semana o aumento correspondeu a $40,41 \%$ e na vigésima quarta semana o aumento caiu para $35,65 \%$, comparados ao período pré-gravídico. A Fig. 5(b) mostra o comportamento do fluxo sanguíneo no arco da aorta, em que ocorre um aumento percentual de $32,28 \%$ na oitava semana, de $47 \%$ na décima sexta e $41.98 \%$ na vigésima quarta semana. Já na aorta abdominal, apresentada na Fig. 5(c) os aumentos correspondem a $62,73 \%$ na oitava semana, $85,58 \%$ na décima sexta e 78,78\% na vigésima quarta. Por fim, a Fig. 5(d) mostra o fluxo na artéria ilíaca interna, na qual na oitava semana 529 


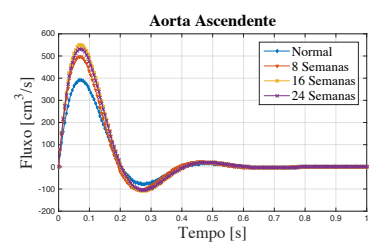

(a) Aorta Ascendente.

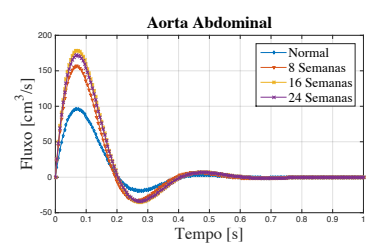

(c) Aorta Abdominal.

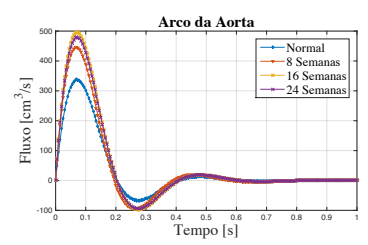

(b) Arco da Aorta.

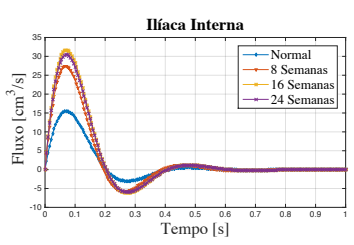

(d) Ilíaca Interna.
Fig. 5. Fluxos sanguíneos em diferentes artérias e períodos.

o aumento percentual corresponde a 77,02\%, 104,37\% na décima sexta e $96.65 \%$ na vigésima quarta semana. Esses valores altos se justificam pelo fato da ilíaca interna ser a artéria mais próxima a placenta entre as analisadas na árvore arterial, sendo também aquela que dá origem à artéria uterina, responsável pela irrigação do útero.

A Fig. 6 mostra o ganho do canal nos quatro períodos analisados, na qual o canal utilizado é o caminho percorrido pelo fluxo sanguíneo saindo do coração até chegar na placenta. Dessa forma, foram analisadas apenas as artérias que pertencem a esse caminho, numeradas de acordo com a Fig. 2 e para fins de comparação, foi feita uma interpolação que resultou nas quatro curvas obtidas. Pelo comportamento das curvas geradas, pode-se notar que ocorre uma atenuação ao decorrer do caminho, gerada pela diminuição do fluxo ao se espalhar pela arvore arterial. $\mathrm{O}$ ganho do canal cai de $0 \mathrm{~dB}$ para $-13 \mathrm{~dB}$ com a propagação dentro do arvore das artérias, e demonstra os efeitos desvanecedores do canal baseado na distancia de propagação. Entretanto, houve um ganho considerável na oitava semana de gestação, em relação ao período pré-gravídico, porém nas semanas seguintes o ganho é mínimo. $\mathrm{O}$ ganho de $2 \mathrm{~dB}$ para longas distâncias percorridas dentro da árvore de artérias em um período de gravidez vem a medida do processo de hipervascularização dentro das 8 semanas. Observa-se também, que a diferença entre as curvas se acentua ao decorrer das artérias que vão se aproximando da placenta, já que no período gestacional o fluxo aumenta com sua formação. O período de maior fluxo de moléculas é a partir das 8 semanas de gestação, onde o ganho do canal ao longo das artérias é maior.

\section{CONCLUSÕES}

As comunicações moleculares permitem estudar sistemas biológicos por meio de teorias de engenharia de comunicações, tornando possível o desenvolvimento de novos sistemas, bem como a compreensão de sistemas já existentes por máquinas biológicas. O foco deste trabalho é analisar os efeitos das adaptações do corpo feminino durante o período gestacional em relação ao sistema circulatório, na forma como o sangue

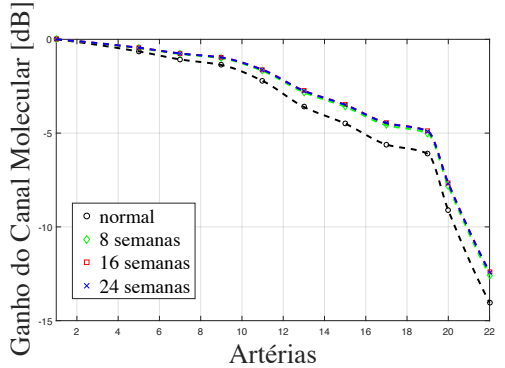

Fig. 6. Ganho do canal molecular ao decorrer do caminho percorrido pelo fluxo do coração à placenta.

é distribuído pelo corpo, e em como isso afeta o ganho do nosso canal molecular, a rede arterial.

Para obter esses resultados, foi calculado o fluxo sanguíneo em toda a rede arterial humana, possibilitando uma análise comparativa do ganho dos canais entre os períodos prégravídico e gestacional. Dessa maneira foi possível observar que o pico máximo ocorreu em torno da décima sexta semana, diminuindo gradualmente até o fim da gestação. Além disso foi observado que o fluxo sanguíneo pode aumentar até em $85,58 \%$ para mulheres grávidas e atingir um ganho de $2 \mathrm{~dB}$ do sinal transmitido. Trabalhos futuros analisarão o percurso e concentração de qualquer partícula invasora na corrente sanguínea, bem como o tempo de propagação necessário até o contato com a placenta em diferentes períodos gestacionais. Isso permitirá que doenças causadas por partículas virais possam ser mais detalhadamente estudadas e que novos sistemas de Entrega Inteligente de Medicamentos possam ser desenvolvidos para mulheres grávidas no futuro.

\section{REFERÊNCIAS}

[1] M. T. Barros, D. P. Martins, and M. S. de Alencar, "Comunicações moleculares: Um novo paradigma de comunicações para aplicações em nanomedicina," Revista de Tecnologia da Informação e Comunicação, vol. 7, no. 1, pp. 31-38, 2017.

[2] M. T. Barros, S. Balasubramaniam, and B. Jennings, "Comparative endto-end analysis of ca 2+-signaling-based molecular communication in biological tissues," IEEE Transactions on Communications, vol. 63, no. 12 , pp. 5128-5142, 2015.

[3] M. T. Barros, "Ca2+-signaling-based molecular communication systems: Design and future research directions," Nano Communication Networks, vol. 11, pp. 103-113, 2017.

[4] N. A. Abbasi, D. Lafci, and O. B. Akan, "Controlled information transfer through an in vivo nervous system," Scientific reports, vol. 8, no. 1, p. 2298, 2018.

[5] Y. Chahibi, M. Pierobon, S. O. Song, and I. F. Akyildiz, "A molecular communication system model for particulate drug delivery systems," IEEE Transactions on Biomedical Engineering, vol. 60, no. 12, pp. 3468-3483, Dec 2013.

[6] J. D. Picon and A. M. P. O. A. de Sá, "Alterações hemodinâmicas da gravidez," Revista da Sociedade de Cardiologia do Rio Grande do Sul, no. $05,2005$.

[7] M. S. Olufsen, C. S. Peskin, W. Y. Kim, E. M. Pedersen, A. Nadim, and J. Larsen, "Numerical simulation and experimental validation of blood flow in arteries with structured-tree outflow conditions," Annals of Biomedical Engineering, vol. 28, no. 11, pp. 1281-1299, Nov 2000.

[8] Â. Baricz, Generalized Bessel functions of the first kind. Springer, 2010.

[9] C.-W. Chen, Y.-W. Shau, and C.-P. Wu, "Analog transmission line model for simulation of systemic circulation," IEEE Transactions on biomedical engineering, vol. 44, no. 1, pp. 90-94, 1997.

[10] K. L. Thornburg, S.-L. Jacobson, G. D. Giraud, and M. J. Morton, "Hemodynamic changes in pregnancy," in Seminars in perinatology, vol. 24, no. 1. Elsevier, 2000, pp. 11-14. 